\title{
Local and global assessment of flotation kinetics
}

\author{
Tomasz Ratajczak ${ }^{1, a}$, Jan Drzymala ${ }^{1}$, and Przemyslaw B. Kowalczuk ${ }^{1}$ \\ ${ }^{1}$ Wroclaw University of Science and Technology, Faculty of Geoengineering, Mining and Geology, Wybrzeze \\ Wyspianskiego 27, 50-370 Wroclaw
}

\begin{abstract}
Most flotation data can be approximated with the first order kinetic equation. However, this equation frequently provides two, not one, parameters, that is the first order flotation rate constant $k$ and maximum flotation recovery $R_{\max }$. Currently, the most often way of evaluation of a set of flotation data is by using efficiency $e_{t 0}=k \cdot R_{\max }$, being the slope of flotation kinetic curve at zero flotation time $(t=0)$. This parameter has a local character and is useful only in very limited cases. It was proposed in this work to use a global flotation kinetic efficiency $(e)$ which can characterize the whole set of kinetic curves. For the considered in this work flotation data, a simple relation $R_{\max }=e \cdot k$ was used. For other sets of flotation data, the global flotation kinetics efficiency very likely will be represented by another equation with the one-adjustable parameter.
\end{abstract}

\section{Introduction}

Flotation is a very complex process [1-3]. One of its aspects is kinetics [4-13]. The kinetics of process significantly influences the economics of flotation. An extensive review on kinetics of flotation was recently published by Gharai and Venugopal [13]. It shows that there are numerous equations which can be used to delineate the flotation kinetics.

According to many studies, most flotation processes follow the so-called first order kinetic equation [15]:

$$
R=R_{\max }[1-\exp (-k t)]
$$

where:

$R$ - floating material mass recovery, $\%$,

$R_{\max }$ - maximum flotation recovery, \%,

$k$ - first order rate constant, $\mathrm{s}^{-1}$,

$t$ - flotation time, s.

Figure 1 shows the flotation data of $\mathrm{Ni}$ et al. [16]. As seen from Fig. 1a, the first order kinetics provides the best approximation of the investigated flotation data.

\footnotetext{
${ }^{a}$ Corresponding author: tomasz.ratajczak@pwr.edu.pl
} 


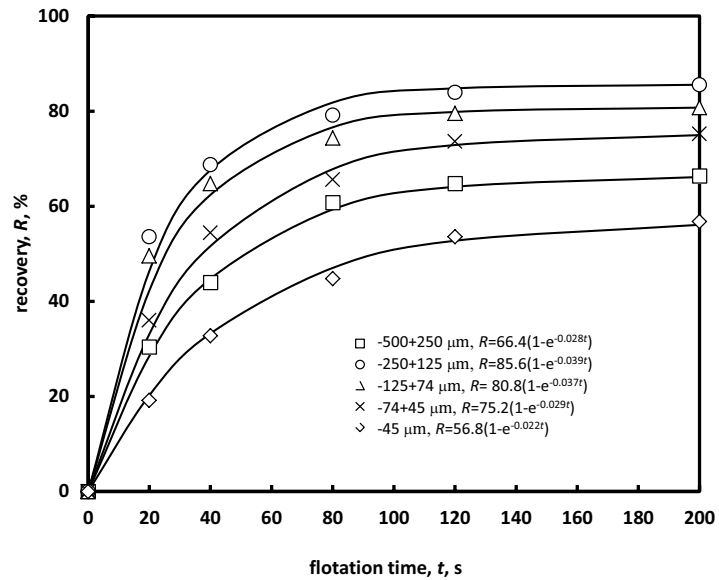

(a)

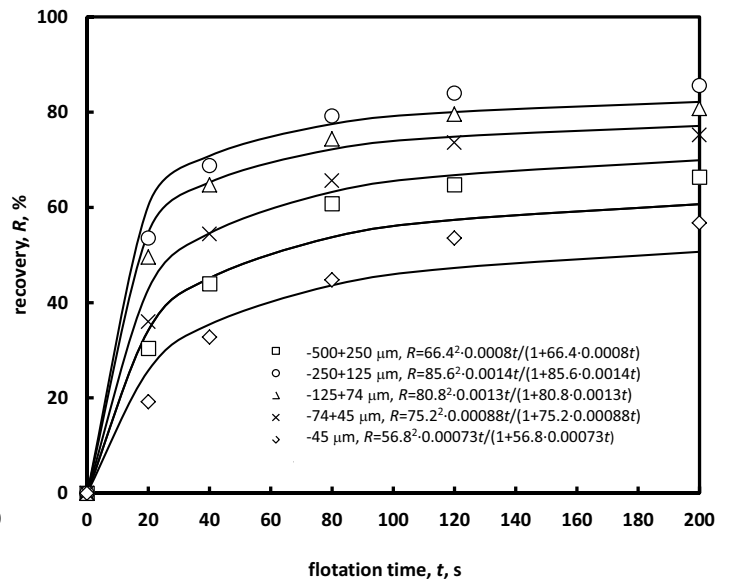

(b)

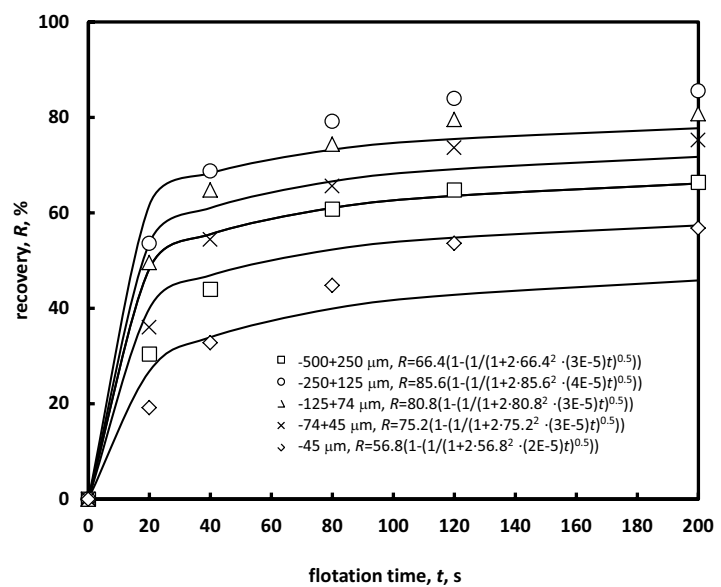

(c)

Figure 1. Kinetics of flotation of different size fractions of coal. Date points taken from Ni et al. [16]. The best approximation of flotation data is provided by the $1^{\text {st }}$ order kinetic equation (a) in comparison to $2^{\text {nd }}$ (b) and $3^{\text {rd }}$ orders (c).

As predicted by Eq. 1, very often the final recovery of floating material is not $100 \%$ (Fig. 1). It complicates the analysis of flotation kinetics because one has to deal with not one but two variables, that is $R_{\max }$ and $k$ simultaneously. As a result, there are many approaches to evaluate the flotation kinetics when $R_{\max }$ is less than $100 \%$. Two of them seem to be the most useful and will be discussed in this paper.

\section{Local efficiency of flotation kinetics}

The first order kinetic equation can be differentiated to create new parameters which can be used to characterize the kinetics of flotation at the certain time. Obviously, such efficiency will be only local, that is valid for a specific flotation time. The well-known approach is the slope of the flotation kinetic plot $R=\mathrm{f}(t)$ at the start of the process, that is at zero time $(t=0)$. This approach was proposed for the first time perhaps by Agar et al. [6], and next used in many investigations [12, 17-21]. The differential of Eq. 1 


$$
\frac{\mathrm{d} R}{\mathrm{~d} t}=\left[R_{\max } \cdot(1-\exp (-k t)]^{\prime}\right.
$$

provides:

$$
\frac{\mathrm{d} R}{\mathrm{~d} t}=\left(-R_{\max }\right) \cdot(-k) \cdot \exp (-k t)
$$

which at $t=0$ assumes the form:

$$
\left.\frac{\mathrm{d} R}{\mathrm{~d} t}\right|_{t=0}=R_{\max } \cdot k .
$$

Thus, the efficiency of flotation kinetics by this approach is given by the formula:

$$
e_{t \mathrm{o}}=k \cdot R_{\max }
$$

where $e_{t \mathrm{o}}$ is the efficiency of flotation, or in our nomenclature, the local flotation kinetics efficiency at $t=0$.

It is obvious that the local efficiency of flotation kinetics can be calculated for any other flotation time. When $t \rightarrow \infty$, then:

$$
\left.\frac{\mathrm{d} R}{\mathrm{~d} t}\right|_{t \rightarrow \infty}=e_{t_{\infty}}=0
$$

while for the case when one chooses to determine the local efficiency of flotation kinetics at the time when the flotation recovery reaches $50 \%$ of $R_{\max }$ the differential is:

$$
\left.\frac{d R}{d t}\right|_{t=1 / 2}=e_{t_{1 / 2}}=R_{\max } \cdot k \cdot \exp (-0.5 \cdot k) .
$$

Figure 2 shows the graphical representation of the local efficiencies of flotation kinetics.

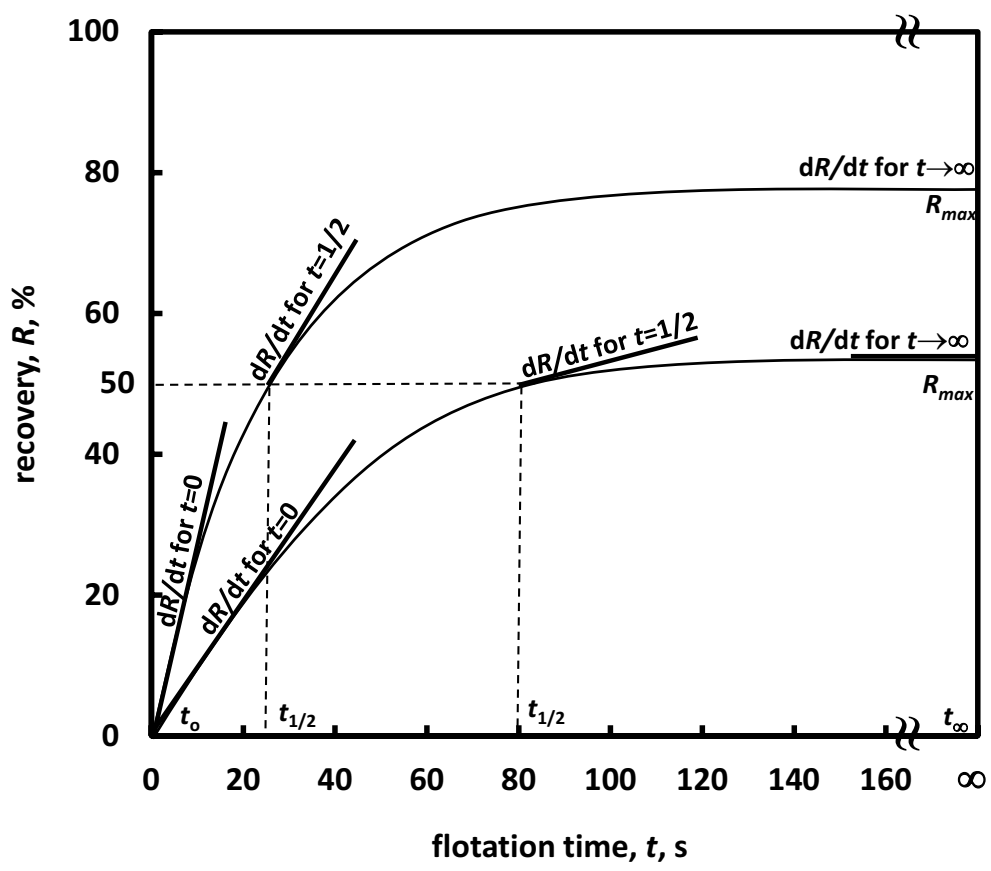

Figure 2. Graphical representation of local efficiencies of flotation kinetics in the form of a derivative $\mathrm{d} R / \mathrm{d} t$ (slope) of the first order kinetic curve at $t=0, t=1 / 2$, and $t=\infty$ (theoretical data). 
Unfortunately, the local approach is not useful because it does not allow to compare two different flotation first order kinetic curves. The shortcomings of the local efficiency of flotation kinetics is given in Fig. 3. Figure 3 shows that when two curves intersect, then the efficiency $e_{t o}$ is misleading because curve $a$ delineates slower flotation kinetics which reaches higher maximum recovery $R_{\max }$, while curve $b$ represents faster flotation kinetics with lower $R_{\max }$. Therefore, one does not know which flotation, in the light of the $e_{t 0}$, is more efficient.

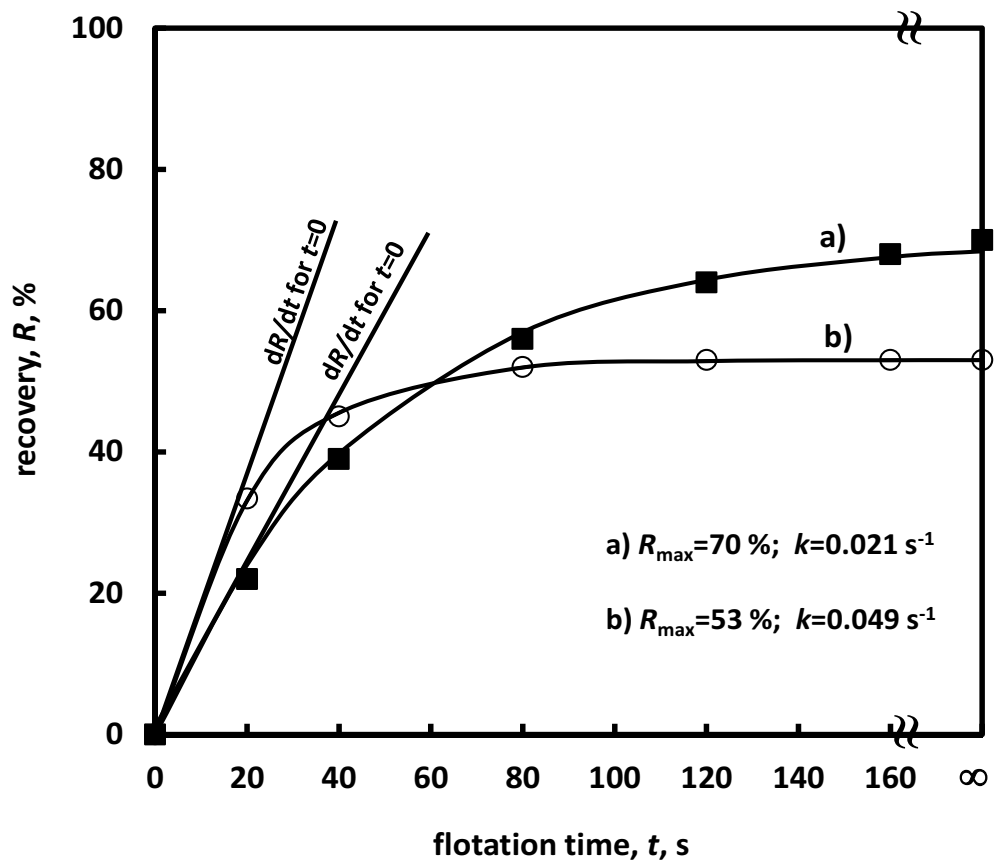

Figure 3. Kinetics of flotation and derivative $e_{t \mathrm{o}}=\mathrm{d} R / \mathrm{d} t$ at $t=0$. Curve $a$ delineates slower and higher flotation $(k$ $\left.=0.021 \mathrm{~s}^{-1}, R_{\max }=70 \%\right)$, while curve $b$ represents faster and lower flotation $\left(k=0.049 \mathrm{~s}^{-1}, R_{\max }=53 \%\right)$.

\section{Global efficiency of flotation kinetics}

For the purpose of comparison of different flotation systems, when both follow the first order kinetic equation and have different $R_{\max }$ and $k$ values, another approach is needed. The most obvious seems to be a relation between $R_{\max }$ and $k$, which has been used by many authors $[13,20]$. However, a next step must be undertaken to find a relationship between $R_{\max }$ and $k$ that can provide a parameter, which can be used to characterize the kinetics of the whole flotation system. Such a parameter can be called the global flotation kinetics efficiency. Figure 4 shows an attempt to determine the global flotation kinetics efficiency for a set of flotation experiments. The experimental data were adopted from Ni et al. [16]. As seen from Fig. 4, the relationship between $k$ and $R_{\max }$ can be approximated with a linear equation having only one-adjustable parameter $e$, which is the global flotation kinetics efficiency of the considered system. For the data presented in Fig. 4 the equations is:

$$
R_{\max }=e \cdot k, R_{\max }=2315.2 \cdot k .
$$

It is expected that for other sets of flotation data the global flotation kinetics efficiency $e$ will be different, and in some case, other than $e=k / R_{\max }$. The one-adjustable parameter equation will be needed to delineate precisely such kinetic data. 


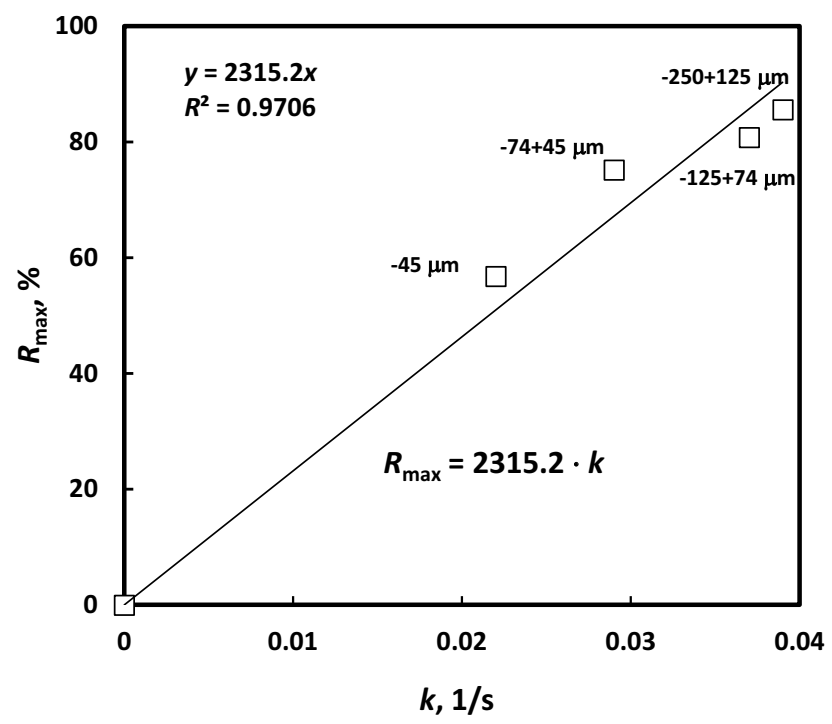

Figure 4. Relationship between $k$ and $R_{\max }$ for kinetics of flotation of different size fractions of coal. Data points from $\mathrm{Ni}$ et al. [16]. The slope of the approximation line is the global flotation kinetics efficiency $e$.

\section{Summary}

The most useful and meaningful way of characterization and evaluation of flotation kinetics seems to be through the so-called global flotation kinetics efficiency parameter. It combines both the first order flotation rate constant $k$ and maximum flotation recovery $R_{\max }$. For the examined here set of flotation data the global flotation kinetics efficiency $e$ was expressed by a simple relation $R_{\max }=e \cdot k$. For other sets of flotation data, the global flotation kinetics efficiency very likely will be represented by another equation, but this requires a further study. The currently often used local flotation kinetics efficiency $e_{t o}$, being a slope of the flotation kinetic curve at $t=0$, is useful only in very limited cases.

\section{Acknowledgements}

This work was partially financed by the Polish Statutory Research Grant 0401/0124/16.

\section{References}

1. A. M. Gaudin, Flotacja (Wydawnictwo Slask, Katowice, 1963)

2. V. I. Klassen, V. A. Mokrousov, An introduction to the theory of flotation (Butterworths, London, 1963)

3. B. Wills, T. Napier-Munn, Wills' Mineral Processing Technology. $7^{\text {th }}$ ed. (ButterworthHeinemann, London, 2006)

4. H. G. Zuniga. Bol. Soc. Nac. Min., Santiago, 47, 83 (1935)

5. T. M. Morris, Tr. AIME, 193, 794 (1952)

6. G. E. Agar, R. Stratton-Crawley, T. J. Bruce, Int. J. Miner. Process., 73, 824 (1980)

7. N. Ahmed, G. J. Jameson, Miner. Process. Extr. Metall. Rev., 5, 77 (1989)

8. M. Brozek, A. Mlynarczykowska, Physicochem. Probl. Miner. Process., 40, 31 (2006)

9. M. Brozek, A. Mlynarczykowska, Physicochem. Probl. Miner. Process., 41, 51 (2007) 
10. M. Brozek, A. Mlynarczykowska, Wydaw. Insty. Gospo. Suro. Mine. i Ene. PAN (Krakow, 2009)

11. O. Guven, M. S. Celik, W. Drelich, Miner. Eng., 79, 125 (2015)

12. A. Bakalarz, P. Nowak, M. Duchnowska, A. Konieczny, E. Kasinska-Pilut, W. Pawlos, R. Kaleta, P.B. Kowalczuk, J. Drzymala, A. Luszczkiewicz, Czasopismo CUPRUM, 2, 75 (2015)

13. Y. Xing, X. Gui, J. Liu, Y. Cao, Y. Zhang, S. Li, Physicochem. Probl. Miner. Process., 52(2), $703(2016)$

14. M. Gharai, R. Venugopal, An. Int. J. 37, 2, (2016)

15. N. Arbiter, C.C. Harris, In: D. W. Fuerstenau (Ed.), Froth flotation (AIME, Ann Arbor, MI, 215, 1962)

16. Ch. Ni, G. Xie, M. Jin, Y. Peng, W. Xia, Powder Technol., 292, 210 (2016)

17. M. Xu, Miner. Eng., 11, 33 (1998)

18. R. Sripriya, P. V. T. Rao, B. R. Choudhury, Int. J. Miner. Process., 68, 109 (2003)

19. S. Muganda, M. Zanin, S. R. Grano, Int. J. Miner. Process., 98, 182 (2011)

20. D. Chipfunhu, M. Zanin, S. Grano, Miner. Eng., 24, 50 (2011)

21. D. Chipfunhu, M. Zanin, S. Grano, Chem. Eng. Res. Des., 90, 26 (2012) 